\title{
Beyond the Art of BART: Bilateral Affective Reprocessing of Thoughts as a Dynamic Model for Psychotherapy Across the Lifespan
}

Arthur G. O'Malley

CAMHS Psychiatrist, Mascot Child and Family Services Ltd, 70 Whitehurst road, Heaton Mersey, Stockport, SK4 3NZ, United Kingdom

\section{Abstract}

This paper sets out to discuss the neurobiological underpinnings of BART psychotherapy which is a new therapy developed by the author over the last 8 years and which is used primarily to treat traumatic stress and dissociative disorders across the lifespan. It begins by presenting a brief overview of BART psychotherapy. It then sets out key historical descriptions of the autonomic nervous system from Darwin in 1872 down to Porges in the 1990s. Porges' Polyvagal theory of safe, unsafe and life threatening environments has made a key contribution to the development of BART. BART also draws on the neuroscience model of genomics and proteonics developed by Prof Ernest Rossi. It moves on to describe some neurobiological foundations that have contributed to the development of BART psychotherapythese include neuroception, brainstem evolution and in utero and early infant development. The use of BART psychotherapy in patients with post traumatic stress disorder is explained in terms of both a myelinated and unmyelinated vagal response. The hierarchical processing dynamics of the autonomic nervous system are described. In this context, the author's quintessential model of the brain is introduced. The different types of information processing relating to trauma are explained. This includes the interplay between top-down and bottom-up processing as described (indifferent ways) by Prof Panksepp and Prof Adolphs, both of which are discussed in relation to the proposed mechanism of action of BART psychotherapy. The links between trauma, attachment and dissociative disorders are explained using the BART model of altered levels of arousal and dissociation linked to the window of affect tolerance, emotional regulation and stability (acronym WATERS). This is complemented by the use of allied acronyms RAPIDS (racing thoughts affective dysregulation, partitioned personality, impulsivity, distress and Suicidality) and FROZEN (freeze reaction, oblivious, zonked, emotionally numb). In a therapeutic context, these are accompanied by the use of visual descriptions are easily understood by patients and clients experiencing these symptoms. Areas into which the theory and practice of BART psychotherapy might be extended are discussed. In particular research might usefully look at recently discovered links between the human immune system and central nervous system. This includes information. The author has included some feedback from patients who have been treated with BART psychotherapy to indicate some of the profound changes that occur following treatment. Some general conclusions are drawn from this feedback and preliminary results of treated families are outlined.

\section{Overview of BART Psychotherapy}

BART psychotherapy is novel therapeutic approach developed over the last eight years by the author. During that time it has been used in a traumatic stress clinic in a Child and Adolescent Mental Health Service and in a parental and infant mental health clinic based in an adult inpatient ward in a district general hospital. The author did initial training in the principles of cognitive behavioural therapy, systemic family therapy and psychodynamic therapy during higher specialist training. As a consultant in Child and adolescent psychiatry he underwent further training in trauma-focused approaches including EMDR, sensorimotor psychotherapy and mindfulness. His passion was to seek the common effective components of all these approaches and describe their neurobiological underpinnings. The result is BART. This is a dynamic model for psychotherapy, which is driven by the patient and is enhanced by the development of rapport within the client-clinician relationship. Each letter conveys a specific component of the psychotherapeutic approach:

B: Bilateral stimulation integrates sensory information from the gut to heart to brain

A: Affective the awareness of emotion is key to resolving traumatic experiences

$\mathrm{R}$ : Reprocessing of information from both the bottom up and the top down is key (p69) $3867 / 2016 / 118$

\section{Publication History:}

Received:September 20, 2016

Accepted: October 29, 2016

Published: November 01, 2016

\section{Keywords:}

\section{BART psychotherapy,} Thalamocortical binding, Polyvagal theory, Information processing, Hyperarousal, Hypoarousal, Dissociation, Evolution of mammalian nervous system, Neuro-immune communication, Quintessential mind session, as there is an opportunity and thinking space for verification, reappraisal and reintegration of any adverse events.

\section{Historical Descriptions of the Autonomic Nervous System}

In 1872, Darwin [1] recognised the dynamic bidirectional relationship between the heart and the brain:

...When the heart is affected, it reacts on the brain... and the brain reacts through the vagus nerve on the heart so there will be mutual action and reaction between these two most important bodily organs.

In 1921 John Newport Langley [2] wrote a book entitled "The Autonomic Nervous System". In it, he described a purely motor system,

"Corresponding Author: Dr. Arthur G. O'Malley, CAMHS Psychiatrist, Mascot Child and Family Services Ltd, 70 Whitehurst road, Heaton Mersey, Stockport, SK4 3NZ, United Kingdom; E-mail: artmail@doctors.org.uk

Citation: O'Malley AG (2016) Beyond the Art of BART: Bilateral Affective Reprocessing of Thoughts as a Dynamic Model for Psychotherapy Across the Lifespan. Int J Psychol Behav Anal 2: 118. doi: https://doi.org/10.15344/2455-

Copyright: () 2016 O'Malley. This is an open-access article distributed under the terms of the Creative Commons Attribution License, which permits unrestricted use, distribution, and reproduction in any medium, provided the original author and source are credited. 
not linked to the brain. Then in 1949, Walter Hess [3] won the Nobel Prize for medicine/physiology. He described how the autonomic nervous system was a paired antagonism system of the internal visceral organs, involving sympathetic and parasympathetic innervations. This idea is based on the evolution of our nervous system over time.

Social behaviour was noted to be a uniquely mammalian feature and was linked to the distribution of nerves to the heart and muscles of facial expression. The heart also became recognised as an endocrine organ, producing both oxytocin and vasopressin. Reptiles, on the other hand, produce vasotocin (a combination of the previous two hormones). We have therefore evolved a complex integrated nervous system. This has a hierarchical response to challenges from the environment, involving four stages:

- Changes have occurred through evolution, which have involved endothelial heart regulation shifting from older unmyelinated nerve circuits to newer myelinated ones.

- The quest for safety via regulation of metabolic output of the heart.

- The social engagement system for safety became integrated over time.

- A process of neuroception in the cerebral cortex that exhibited downward inhibitory control of the vagus nerve.

This theory evolved during the 1990's when Dr Porges was in discussion with neonatologists involved in caring for very premature infants, [4-7]. They were very susceptible to infection and often succumbed. Dr Porges set out over the next few years to understand the multiple functions of the infant's vagal nerve. The old unmyelinated vagus nerve is designed to preserve metabolic resources. Although initially protective, it can also kill you by causing panic and immobilisation. When Dr Porges was working in a neonatal intensive care unit he found that, in newborns, vagal tone was protective when associated with heart rate rhythmicity. A paediatrician wrote to him stating: "Too much of a good thing is bad," suggesting a vagal paradox in that the vagus could be both protective and lethal. This led Dr Porges to pursue his Polyvagal theory. He discovered that the phylogeny of the autonomic nervous system was not well described. He proposed that in the embryo the vagus nerve had two primary divisions:

1. The dorsal motor nucleus of the vagus nerve, which has its origin in the nucleus tractus solitarius (NTS).

2. The ventral motor nucleus of the vagus nerve, which has its origin in the nucleus ambiguus in the brain stem.

The ventral motor nucleus of the vagus nerve controls the striated muscles of the face and head, the larynx, the pharynx and the common nuclei of the facial and trigeminal cranial nerves. Thus the innervation of the heart goes with that of the head as they are wired together form early embryonic development. This provides evidence for the theoretical underpinning of The Art of BART: Bilateral Affective Reprocessing of Thoughts as a dynamic model for psychotherapy pioneered by O'Malley, [8].

The body's hierarchical response to an environmental challenge is initially regulated by the ventral nucleus of the vagus nerve, in its control of the face and heart. Pre-term babies are vulnerable to this challenge, as their facial muscles do not fully work at birth. Also their sensorimotor system will register that they are in an unsafe state. The main mode of change is to involve neuro-regulation of the periphery by the brain. BART psychotherapy starts with stimulation of the peripheral nervous system at the level of the gutbrain and heartbrain, before reprocessing at a headbrain level. It is my hypothesis that this helps to rewire faulty neural networks associated with traumatic events during pregnancy, infancy, and childhood throughout the lifespan. This is supported my comments of patients who describe how negative associations with traumatic memories are no longer accessible following treatment. It is my research proposal to explore this hypothesis with fMRI scans before and after treatment to assess any changes in activity of the relevant neural networks.

Dr Porges' Polyvagal theory is also consistent with John Hughlings Jacksons' [9] theory of dissolution of the autonomic nervous system. When humans are in a safe environment, visceral homeostasis promotes growth and restoration. The myelinated vagus acts on the cardiac heart rate, slowing the heart and inhibiting the fight-or-flight mechanism of the sympathetic nervous system (SNS). It also reduces cortisol secretion from the hypothalamicpituitary-adrenal axis and decreases immune mediated inflammation by decreasing circulating cytokines. Throughout the process of evolution and natural selection, the brain stem nuclei of the nucleus ambiguus merged with those the face and head. An integrated social engagement system then emerged.

To ensure survival in dangerous or life threatening situations, further evolution of the nervous system was necessary. This involved progressive recruitment of the SNS and behaviour associated with activation the dorsal motor nucleus of the vagus nerve, (DMX), such as freeze, fall and feigned death. Normally the vagus nerve acts as a brake on the body's heart rate. The resting heart rate is $70-85$ beats per minute. If this vagal brake is released, e.g. by being cut, the heart rate goes up to $100-110$ beats per minute. In reality this occurs every time we suddenly stand up and scan the environment. Our heart rate is therefore regulated by these distinct phylogenetic systems. For social engagement to occur, we must first assess risk in the environment and inhibit instinctive brainstem and limbic system control of our fight, fright or flight and freeze responses.

\section{Some Principles of Affective Neuroscience, Which are Foundations of BART Psychotherapy}

The nervous system is continuously evaluating risk via an unconscious process, which Dr Porges has called Neuroception. This helps us to distinguish safe, dangerous and lifethreatening situations from environmental cues. The key brain areas thought to be involved in this process are the temporal cortex, amygdala and periaqueductal grey matter. Together with activation of the social engagement system we are able to interpret voices, facial expression and hand movements or gestures. The relationship between the cortex brainstem and cranial nerves is illustrated in Figure 1. The cranial nerves are activated by bilateral stimulation (BLS) of the peripheral nervous system. In the Art of BART psychotherapy, I often place tactile units at the level of the ankles and hands or wrists. This provides a stimulus to the superior colliculi in the brainstem. As well as the cranial nerves acting on the structures below, the oculomotor trochlear and abducens nerves also have their original nuclei at this level of the brainstem.

This comprises a somatomotor component (i.e. special visceral efferent pathways that regulate the muscles of the head and face shown as solid arrows) and a visceromotor component (i.e. the myelinated vagus that regulates the heart and bronchi shown as dashed arrows). 
Citation: O'Malley AG (2016) Beyond the Art of BART: Bilateral Affective Reprocessing of Thoughts as a Dynamic Model for Psychotherapy Across the Lifespan. Int J Psychol Behav Anal 2: 118. doi: https://doi.org/10.15344/2455-3867/2016/118

Page 3 of 15

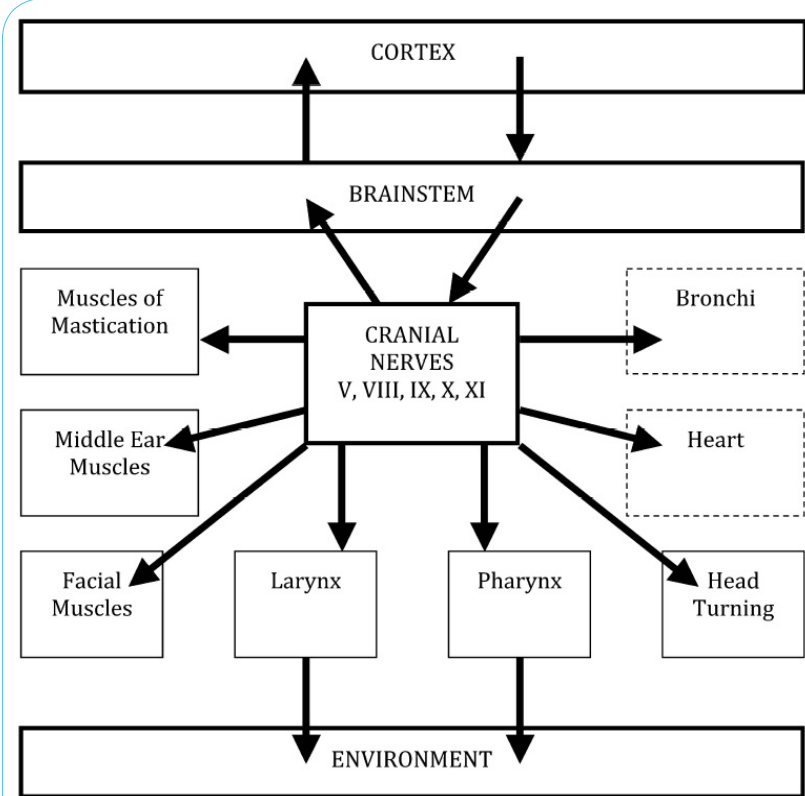

Figure 1: Relationship between the cortex, brainstem, cranial nerves and the environment.

The nucleus tractus solitarius has an integral role in controlling breathing and hence optimising the interaction with the lung parenchyma and airways. Activation of the NTS during BART psychotherapy should help in management of patients with anxiety disorders. Of note the primitive unmyelinated pathways of the vagus nerve mediate this pathway.

The three stages of reaction predicted by the Polyvagal theory of Dr Porges are:

The ventral vagal complex (VVC). These fibres originate in the nucleus ambiguus and control larynx, pharynx, bronchi, oesophagus and heart. It is involved in control and coordination of sucking, swallowing and vocalization with breathing. In treating traumatic stress with BART psychotherapy the involvement of cranial nerves $\mathrm{V}$, VII, IX, X and XI should be assessed, as they are first to be affecting by involvement of the VVC.

The sympathetic nervous system (SNS). This prepares the body for mobilization but is activated by stress and high levels of arousal. The label sympathetic links to its historical association with feelings in contrast to the parasympathetic nervous system, which reflects its function to protect the body against overwhelming feelings.

The dorsal vagal complex (DVC). This is involved in digestion, taste and protection from hypoxia. The neuronal basis is the connection between the nucleus tractussolitarius and the dorsal motor nucleus of the vagus nerve. Critically this is the basis of reptilian vagal control of the heart and lung. Cold-blooded reptiles can survive an environment depleted in oxygen. However the human brain has twenty percent of the body's metabolic requirement for oxygen and quickly becomes vulnerable when the dorsal motor nucleus of the vagus nerve is activated. This occurs in complex trauma leading to dissociation. The patient needs to be maintained within their window of affect tolerance and emotional regulation. The unmyelinated fibres of the dorsal vagus [10] are believed to trigger bradycardia.
Neural and Neuropeptide regulation of the dorsal vagal complex in a safe environment. It includes both the nucleus of the tractus solitarius (NTS) and the dorsal motor nucleus of the vagus (DMX). In this safe environment oxytocin (OT) is released centrally to the sensory and motor parts of the dorsal vagal complex as well as systemically to the visceral organs. Oxytocin is crucial in helping to foster a calm anti 'traumatic stress' state. The intense $120 \mathrm{~min}$ therapeutic sessions of BART ensure patients leave in this state.

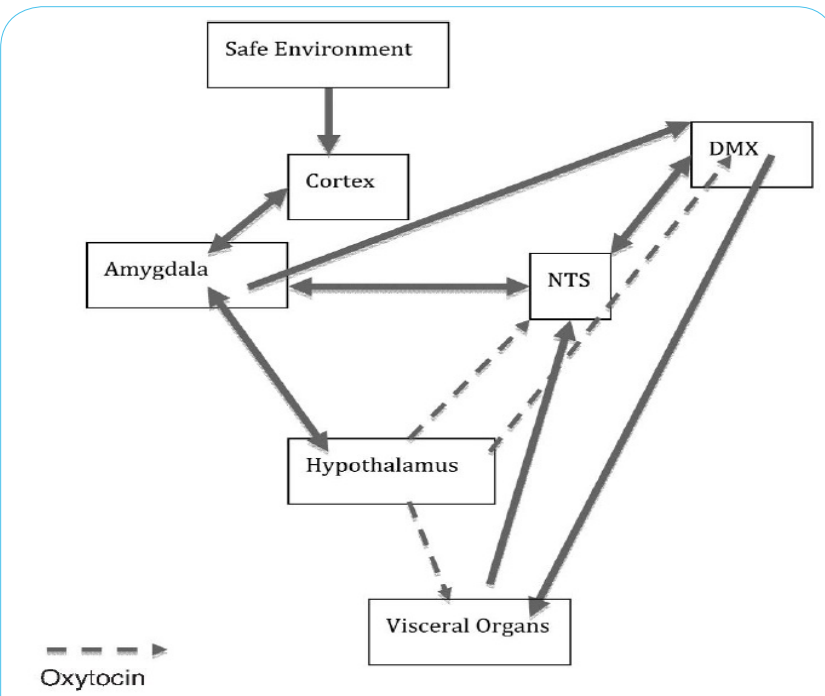

Figure 2: This shows the relationship between the brain and the body when the environment is perceived as safe. Diagram modified by Sorcha O’Malley.

As the person is exposed to an unsafe environment, the neural and neuropeptide regulation of the dorsal vagal complex changes. The mobilization of the sympathetic nervous system triggers arginine vasopressin (AVP) to communicate across the neural pathways from the hypothalamus and the nucleus tractus solitarius. This changes the set point of vagal reflexes such as the baroceptor reflex. This is part of the Jacksonian Dissolution theory, which predicts sympathetic nervous system activation as traumatic stress intensifies. It is essential for the attuned therapist to recognise when their patient feels unsafe

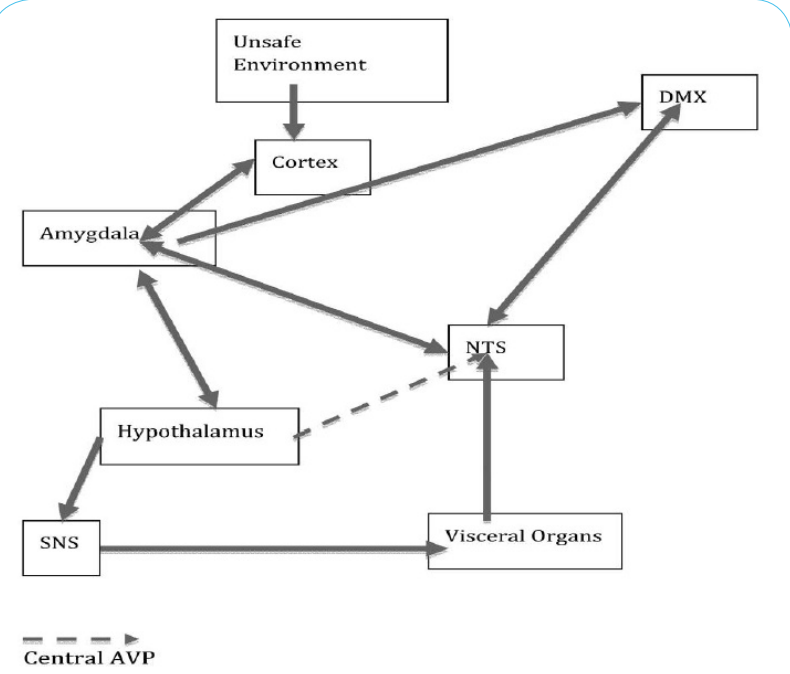

Figure 3: This schematic diagram illustrates the activation of the amygdala and dorsal motor nucleus of vagus nerve when the patient or clients' cerebral cortex is exposed to an unsafe environment. Diagram modified by Sorcha O'Malley 
Citation: O'Malley AG (2016) Beyond the Art of BART: Bilateral Affective Reprocessing of Thoughts as a Dynamic Model for Psychotherapy Across the Lifespan. Int J Psychol Behav Anal 2: 118. doi: https://doi.org/10.15344/2455-3867/2016/118

Page 4 of 15

Again in a life-threatening environment the neural and neuropeptide regulation of the dorsal vagal complex exposes the most primitive parts of our reptilian nervous system to become activated. The previous fight-flight responses of the sympathetic nervous system are deactivated. The immobilization fear-freeze response becomes predominant. The DMX provides a vagal surge to the visceral organs. Peripheral and systemic AVP stimulates visceral afferents via the NTS. Many patients shutdown or dissociate at this stage of therapy.

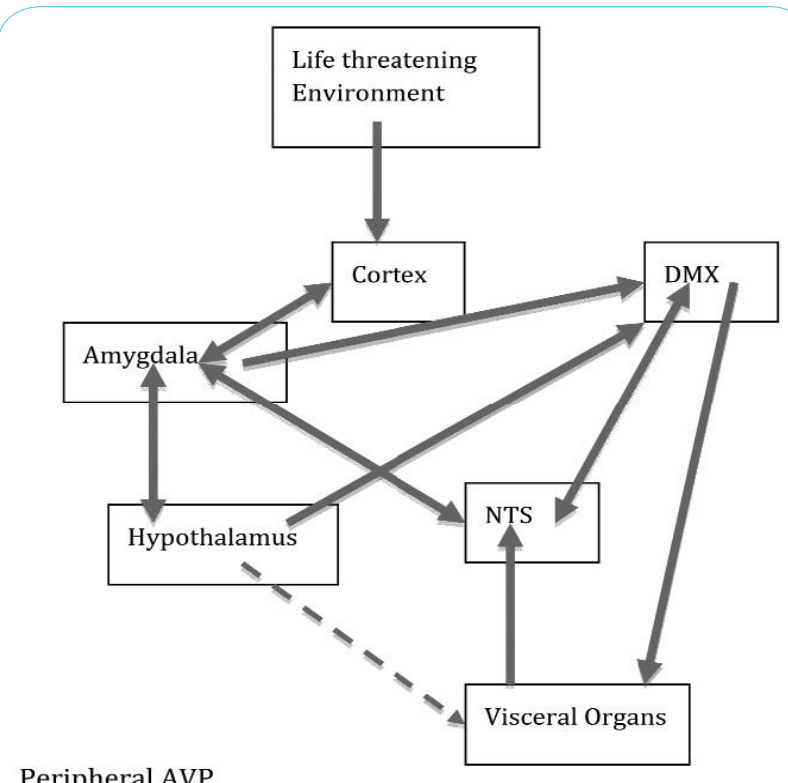

\section{Peripheral AVP}

Figure 4: Schematic diagram shows further activation of dorsal vagus nerve during exposure of the patient or client to a life-threatening environment. Diagram modified by Sorcha O'Malley.

Thus the ANS responds in a hierarchical way when exposed to increasingly unsafe environments. John Hughlings Jackson, [9] proposed the theory of dissolution to explain this phylogenetically based response.

"The higher nervous arrangements inhibit (or control) the lower, and thus, when the higher are suddenly rendered functionless, the lower then rise in activity."

According to Dr Porges this dissolution is an adaptive response to the person's exposure to increasingly challenging environments. The VVC provides the initial response to traumatic stress. This provides the framework for the social engagement system and appropriate body language with facial expressions. As its effects are withdrawn the SNS becomes overactive. Finally the DVC triggers defecation, apnoea, sino-atrial node stimulation leading to bradycardia and vasovagal syncope. During traumatic stress faster acting unmyelinated neuronal circuits become activated. This may help explain some of the poorer outcomes of cog. In my clinical experience over years using BART psychotherapy, bilateral stimulation with buzzers combined with bilateral cerebellar resonance activates the gut instinct and heartfelt sensations to connect with the brainstem nuclei. From here the continuous auditory stimulation adjacent to the mastoid process at a frequency of $40 \mathrm{Hertz}$ (gamma frequency) picks up the information. Patients report a tingling sensation in the forehead in the area of the prefrontal cortex where meaning is made of their experience. They develop an appropriate perspective on the past with the development of insight and often increased self-empathy.
It is my research hypothesis that the continuous use of buzzers held bilaterally for ninety minutes activates the peripheral nervous system. As this signal reaches the cerebral cortex the buzz of thought may permeate the arachnoid granulations leading to the development of new neural networks. This information is integrated and process by DNA and genomics and is communicated back to the body by the glymphatic and immune systems for protection from future adverse 'antigenic' challenges.

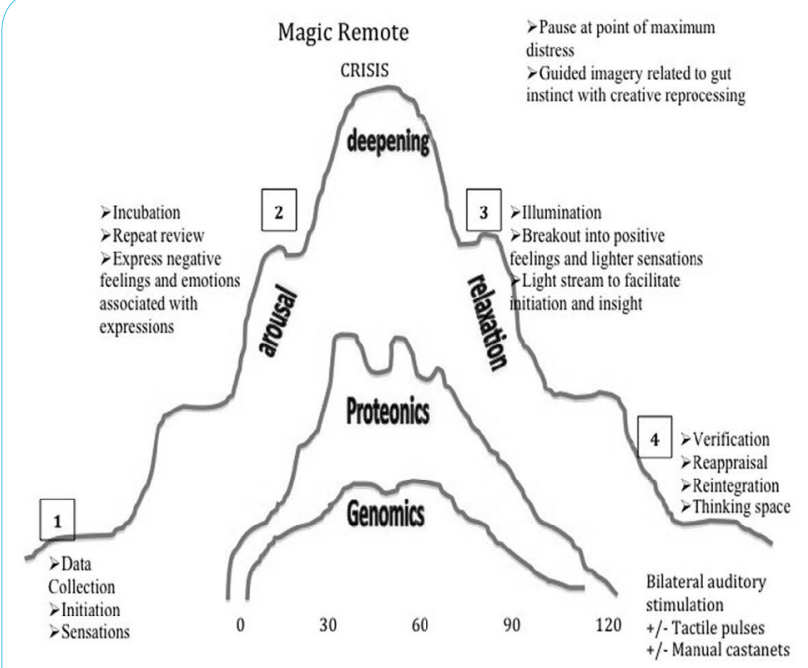

Figure 5: Model of BART psychotherapy session compared to neuroscience model of Rossi. Drawing created by Sorcha O’Malley and adapted from original by Prof Ernest Rossi.

\section{Parasympathetic System}

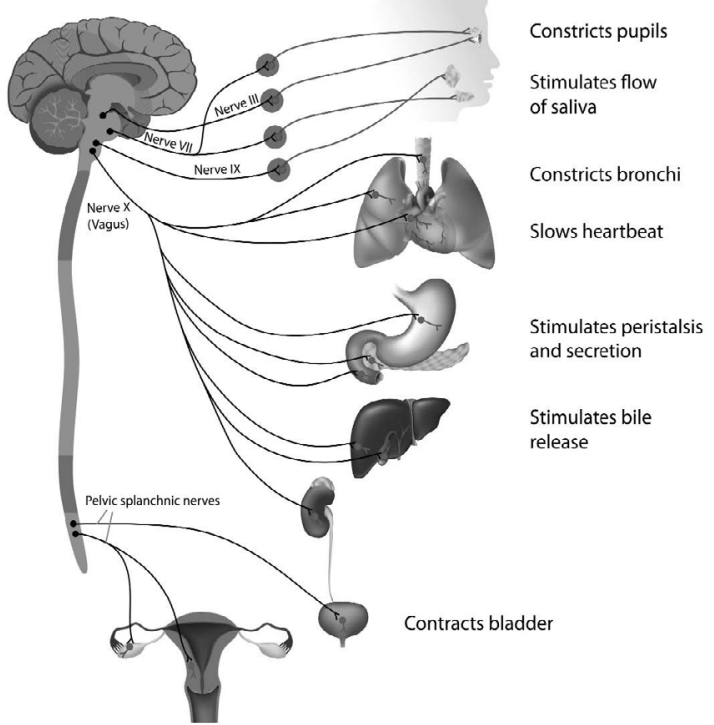

Figure 6: The parasympathetic system illustrates the extent of the path of the vagal nerve in the body. Vagus means wanderer and reflects the tenth cranial nerve's journey from head to lung, heart, liver, stomach, spleen, kidney, small \& large intestine and bladder. The bilateral cerebellar auditory and tactile stimulation during BART psychotherapy activates the nerves and hence the feelings associated with each of these organs where relevant in either trauma resolution or peak performance.

According to Dr Porges' Polyvagal theory, dorsal vagal unmyelinated fibres become activated when the patient or client is exposed to a life-threatening environment. This means that their reptilian brainstem processes all the incoming sensory information. Traumatic 
Citation: O'Malley AG (2016) Beyond the Art of BART: Bilateral Affective Reprocessing of Thoughts as a Dynamic Model for Psychotherapy Across the Lifespan. Int J Psychol Behav Anal 2: 118. doi: https://doi.org/10.15344/2455-3867/2016/118

Page 5 of 15

experiences can become locked in at this level of the brain. During BART psychotherapy I often ask patients or clients to hold bilaterally activated tactile units either side of their shoulders or neck. This has the dual advantage of stimulating bilaterally the origins of several of the cranial nerves processing this traumatic sensory information. Also this allows for activation of the reticular acting system and consequent production of pontine-occipitalgeniculate waves. These are a precursor to REM and towards the end of BART psychotherapy sessions I have observed the majority of patients yawning as if about to enter a REM state. In this mindful state their nervous and motor systems become still enabling effective reprocessing of sensations emotions feelings and thoughts.

A schematic drawing of the dorsal view of the goldfish brain showing the main brain divisions: forebrain (the olfactory bulbs and telencephalon), midbrain (mesencephalon) with the optic lobes, cerebellum, and vagal lobes is shown in Figure 7. The floor of the telencephalon is the evolutionary primitive structure for the basal ganglia in higher vertebrates. The roof of the telencephalon is the precursor to the hippocampus. The optic lobes and the cerebellum have their usual higher vertebrate equivalents. The vagal lobes are the locus of cell bodies for the glossopharyngeal nerve, which is important for taste. The mesencephalon is not visible from a dorsal view and is located at a rostral and caudal position, respectively, underneath the midbrain's optic lobes. The schematic drawing of the lateral view of goldfish brain shows the level of the sections in the lower part of the diagram.

These can be compared with the embryological divisions of the human brain. It is interesting to observe how the vagal lobe of the goldfish has evolved into the human brainstem. This provides further neuroanatomical support for the theoretical validity of BART psychotherapy, where a key consequence of bilateral cerebellar stimulation is activation of the brainstem of the patient or client. Further BART psychotherapy activates neural synapses and often a tingling sensation is experienced, as I believe new synaptic connections are made and axon rewiring occurs. An independent mind finds verbal expression easier as emotional blocks are overcome and creativity flows more freely. Broca's area (expressive language) and Wernicke's area (receptive language) become activated. Blood flow increases in the prefrontal cortex, thus better transmission of information is possible.

The periaqueductal grey is an area of grey matter surrounding the third and fourth ventricles. It plays a key role in mediating the brain's response to trauma. Neuroception has evolved below the level of consciousness. If the nervous system appraises the environment as dangerous when it is actually safe, an inappropriate response can occur. Table 1 illustrates the various responses to safe and dangerous situations:
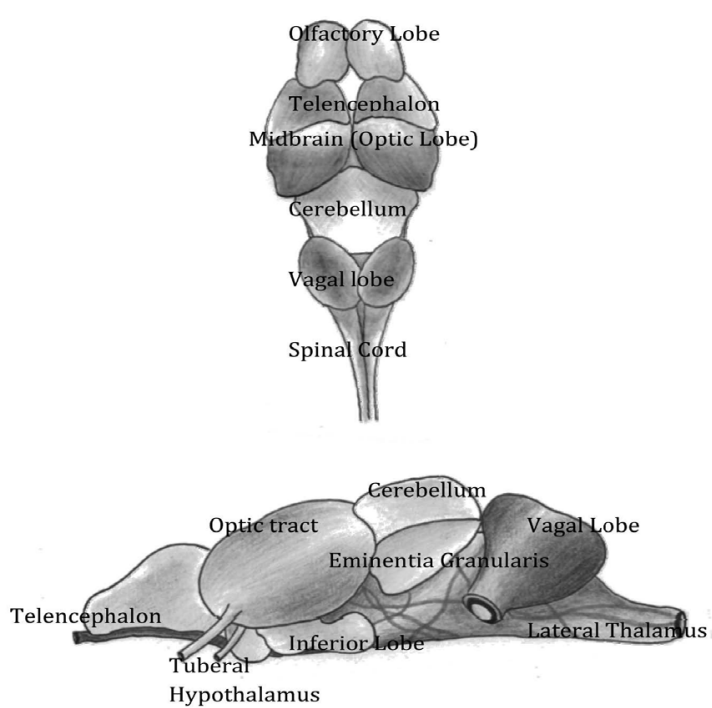

Figure 7: Dorsal views of the goldfish above and lateral view below. Fish are one of the five most well known classes of vertebrates (animals with backbones). These are mammals, birds, fish, reptiles and amphibians. fish and reptiles have minimal cerebral cortex but a well-developed cerebellum and predominant olfactory lobe. This illustrates the ancient phylogeny of these structures, which we have inherited from our reptilian and amphibian ancestors. They have a strong survival instinct driven by their medulla oblongata and brainstem. Drawn by Sorcha O'Malley.

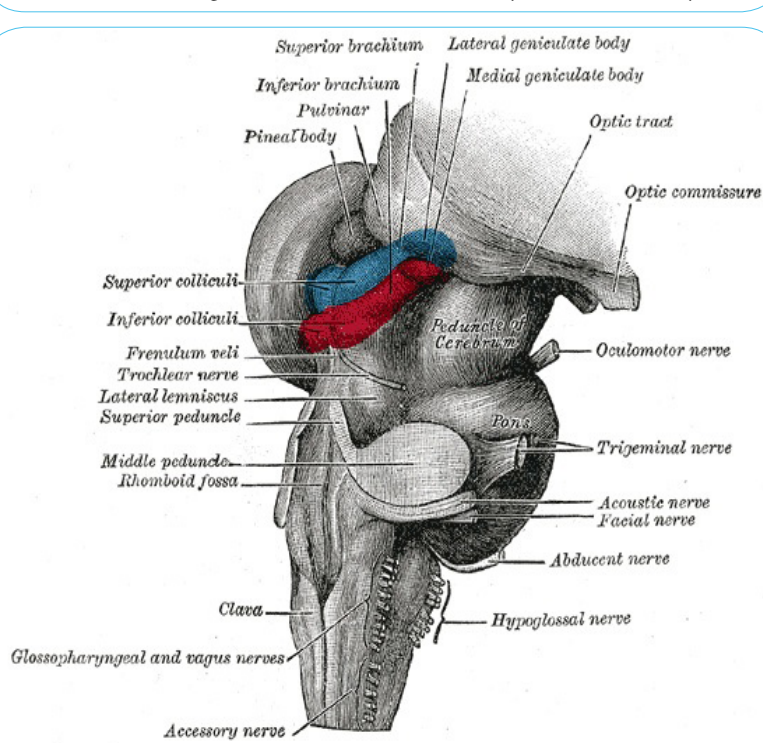

Figure 8: By inverting the reptilian brain by ninety degrees the similarity to our own brain stem becomes clear. Dampening brainstem reactions is a key goal of BART psychotherapy and peak performance. This is facilitated by bilateral cerebellar stimulation, which plays a role in activation of the ascending reticulating activating system in the brainstem.

\begin{tabular}{|l|l|l|l|}
\hline Response & Level of Arousal & Nervous System Activated & Neuro-anatomy Endocrine System \\
\hline Fight & Increased (hyper) & Sympathetic & - H.P.A Axis - Adrenaline \\
\hline Flight & Increased (hyper) & Sympathetic & - H.P.A Axis - Adrenaline \\
\hline Fright & Increased (hyper) & Sympathetic & - H.P.A Axis- Adrenaline \\
\hline Freeze & Decreased (hypo) & Unmyelinated branch of vagus & Post-ganglionic muscarinic nerves -acetylcholine \\
\hline Faint (vaso- vagal syncope) & Decreased (hypo) & Unmyelinated branch of vagus & Acetylcholine \\
\hline Feigned death & Decreased (hypo) & Unmyelinated branch of vagus & Acetylcholine \\
\hline
\end{tabular}

Table 1: Range of responses of the autonomic nervous system to traumatic stress. 
For the SES to work these instinctive responses and gestures must be switched off. This involves a level of top down control from the prefrontal cortex to the bilateral almond shaped amygdalae. When the risk is rated as high in the environment by a patient's neuroceptive processes, both amygdala and the PAG are activated. The PAG's dorsolateral, ventrolateral, and lateral, rostral and caudal nuclei trigger instinctive defensive (passive or active) or escape behaviours. I believe this has major implications for the evaluation of risk in the setting of patients' attending our mental health services.

For a patient or client neuroception at an unconscious level is always evaluating threat or risk and triggering a cascade of visceromotor and somatomotor reactions. If the nurse or therapist assumes these are conscious behaviours the level of intervention is likely to be unsuited to the patient's internal state. In order for the therapist to gauge the neurological level of risk, it is important that a detailed history of development both in utero and infancy is taken. By taking a more detailed traumatic history it is likely that knowledge will be gained about how the patient will have relied upon their primitive or unmyelinated vagal nerve responses during previous episodes of traumatic stress. In these cases forearmed is forewarned and the nurse or therapist's task is to ensure that the patient or client doesn't misconstrue a safe inpatient environment for a perceived life threatening one for example.

The level of myelination of nerve fibres occurs rapidly in a linear fashion from 26-40 weeks of gestation. Thus prematurity severely impairs the myelination process. During the first three months of infancy, further rapid myelination occurs in preparation for bonding between the primary care giver and the infant. From 6 months to 3.5 years of age, attachment patterns are laid down. If the adult patient has had a sufficiently traumatic childhood (or was born with a pervasive developmental disorder), then opportunities for optimal SES development are minimal. The risk of self-harm and harm to others is increased because of this altered neuroception, leading to the misinterpretation of a safe environment as unsafe. The use of seclusion in such a patient may trigger aspects of their PAG. This may stimulate phylogenetically older responses of the dorsal vagal complex (DVC) and cause rapid deterioration in their mental state.

The first three years of brain development primarily involve the development of the right cerebral hemisphere. It processes sensory information, positive affective information and the adaptive expression of negative affect, [11-14]. The right hemisphere also appears to have a primary role in regulating cardiac function, represented by cardiac vagal tone (CVT). This is the sum of the inhibiting influences on the heart of the myelinated vagal pathways i.e. the ventral vagal complex (VVC), originating in the nucleus ambiguus (NA) and the immobilising effects of the dorsal motor complex of the vagal nerve (DMX), originating in the nucleus tractus solitarius (NTS). The nucleus tractus solitarius is located at the origin of the vagal nerve and has evolved to mediate sensory information from the periphery. This can be represented by the following equation:

\section{$\mathrm{CVT}=\mathrm{VVC}(\mathrm{NA})+\mathrm{DMX}(\mathrm{NTS})$}

I believe that BART psychotherapy represents a unique opportunity to combine the higher frequency of the VVC with the lower frequency bands associated with the DMX. These become integrated with the information processing of the gutbrain, heartbrain and headbrain, enabling the patient to resolve their traumatic experiences.
During the process of BART psychotherapy, attention is paid to heart rate variability, ensuring that neither the VVC nor DMX are overactivated and that the patient remains within their window of tolerance (Figure 11). Afferent feedback from the viscera is emphasised in the early stages of BART psychotherapy by accessing and reprocessing gut reactions. The insular cortex is also thought to be involved in the development of meaning and insight by integration of the diffuse visceral feedback and allowing cognitive awareness and insight or meaning to emerge. This is the goal of the latter stages of BART psychotherapy.

Hobson [17] describes top-down processing as "when we decide we want to go somewhere, we voluntarily initiate movement sequences". Once they begin the sequences are so automatic that they no longer require conscious supervision. Walking, jogging, even sprinting are controlled by brain stem motor pattern generators. We flip a switch in our cortex and our lower brain coordinates the motion because that command centre is in the cortex. Your decision to walk is one of the many examples of top-down processing. The activities of very young children and those experiencing trauma are governed primarily by their sensory motor and their emotional systems, in other words by bottom up processes. A child's job is to explore her world through these systems building the neuro networks that are the foundation for later cognitive development, $[18,19]$. Wired to be governed by somatic and emotional states children respond spontaneously to sensory motor and affective cues. Traumatised people frequently observe how their senses and emotions control them, as they are unable to regulate these functions. For example someone who has experienced trauma will be dominated by the startle reflex and by defensive or orientating responses. The default mode network (DMN) is a pattern of interaction between different parts of the brain that is known to be present at rest. Traumatic stress has been discovered to disrupt this network and prevent normal brain function. This an adult patient with unresolved traumatic stress before ten years of age retains the DMN appropriate to that stage of development, This has major implications for deciding on type of trauma therapy when they present as an adult when their neural networks are functioning at a nine year old level. In is my intention to test the hypothesis that BART psychotherapy can restore maturational development of this and other brain networks. Appropriate brain imaging and electrophysiological studies can test this before and after intensive BART psychotherapy. Firstly linking the mind to the body is the spinal cord with the peripheral nervous system and the connection to the cranial nerves. Secondly at the base of the brain you have the brainstem. This governs most of our instinctual survival responses. Thirdly the limbic system processes and regulates emotional states. Key components are the thalamus and right and left sided amygdalae. Fourthly attached to the brainstem, limbic system and cerebral cortex is the cerebellum, which incorporates emotional and sensory integration as well as large and fine motor functions. Only in recent years has its full significance in regulating thoughts feelings sensations and emotions become known. Known as the little brain it contains more neuronal synapses at birth that the rest of the brain. It is fully matured by the second year of life. Thus it is an ideal container for the somatosensory experiences associated with infancy. In BART psychotherapy the bilateral auditory frequency of 40-60 Hertz is applied adjacent to each cerebellum. This helps to both dampen down brainstem reflexes and communicate directly with $40 \%$ of its neuronal connections to the prefrontal cortex.

Fifthly we have (a) the archo-mammalian or paleocortex, which comes from our small mammalian ancestors e.g. rodents, (b) the neomammalian cortex, which has developed from our largest 
Citation: O'Malley AG (2016) Beyond the Art of BART: Bilateral Affective Reprocessing of Thoughts as a Dynamic Model for Psychotherapy Across the Lifespan. Int J Psychol Behav Anal 2: 118. doi: https://doi.org/10.15344/2455-3867/2016/118

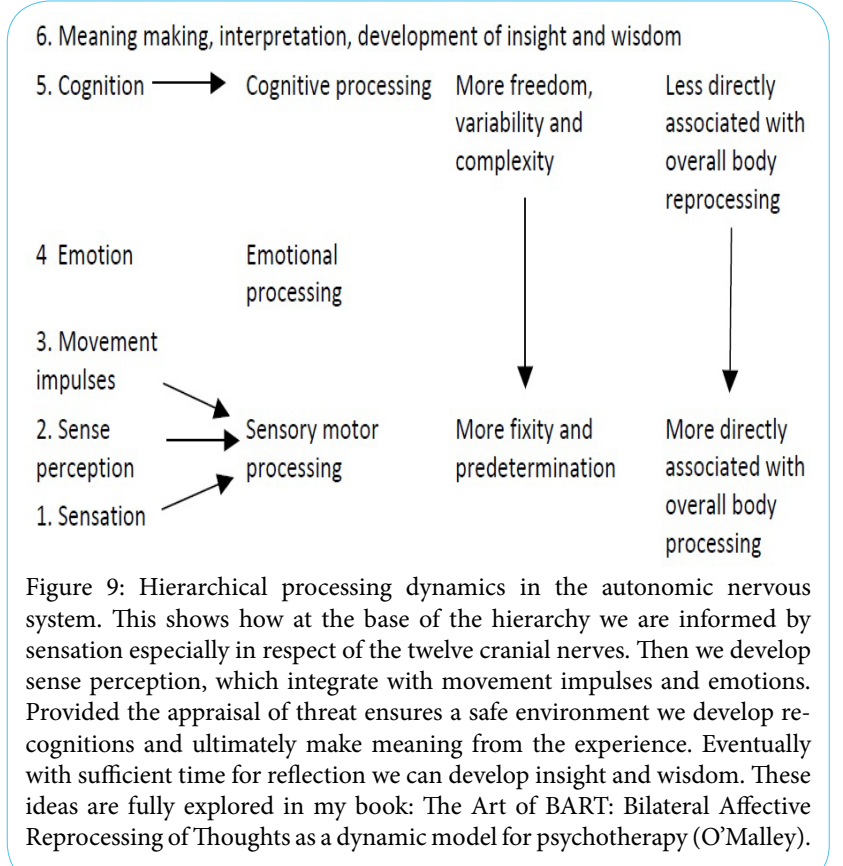

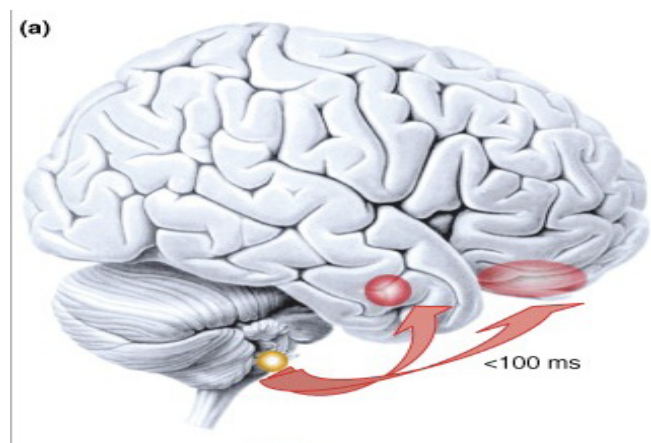

(b)

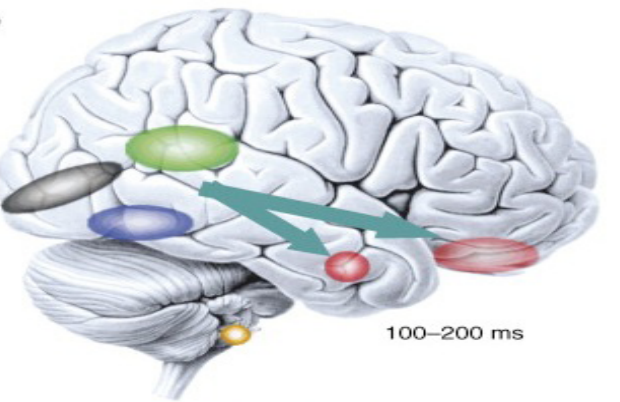

(c)

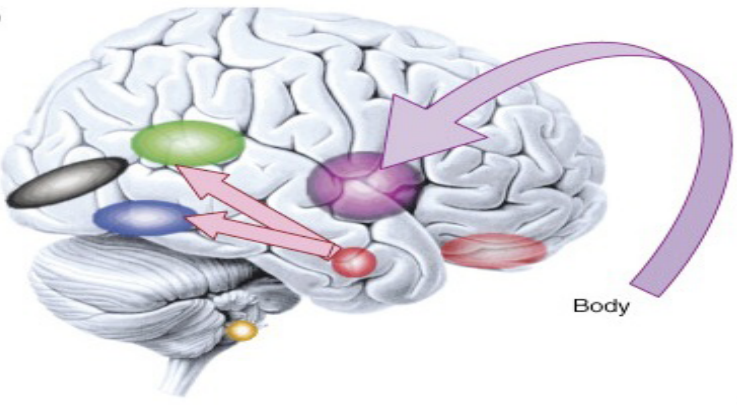

Responses to emotional visual stimuli can be registered in the amygdala and prefrontal cortex due to subcortical input from the peripheral nervous system. This BOTTOM-UP processing lasts from 40$100 \mathrm{~ms}$.

Figure 10: Quintessential Model of the Brain, [20].

At $100-200 \mathrm{~ms}$ the different areas of the sensory cortex provide input to the amygdala. The superior temporal cortex (green) encodes facial expression, while the fusiform gyrus (blue) encodes static information e.g. identity. MIDDLE-LEVEL Processing.

Figure 11: Emotional reprocessing at top down and bottom up levels of the brain. This illustration of the timeline for neuronal processing is reprinted with permission from Professor Ralph Adolphs, [22]. 
mammalian ancestors for example the gorilla. This neomammalian cortex facilitates socioemotional relationships and governs teamwork, taking turns and sharing. Finally we have (c) the prefrontal cortex, which encourages abstract thoughts and processes, arts, language and humour as well as games. In human evolution this now comprises forty percent of the frontal cortex and is the last part of the brain to myelinate and therefore have the capacity for insight and reflective thought. This is built from the bottom up in terms of the phylogenetically oldest to newest parts of the brain. As already explained the oldest most hardwired part of the brain is the brainstem and limbic system, which is approximately 200 million years old. Next up is the cerebellum or little brain. Fully developed by age 2 it contains a greater density of neurons than the rest of the brain. It provides inhibitory input to the brainstem and $40 \%$ of its fibres connect directly to the prefrontal cortex. This gives the cerebellum a crucial role in emotional and cognitive processing. Our smallest mammalian ancestors grew the archeomammalian or paleocortex, which was superceded by our nearest mammalian ancestors who have the surrounding neocortex. Finally only 10,000 years ago we evolved the prefrontal cortex. This provides $40 \%$ of the volume of the cortex and provides us with our uniquely human attributes of language, reflective thought and consciousness. It is the least hardwired part of our brain and the first to go offline as the blood flow is diverted to the brainstem when our survival is threatened during traumatic stress.

Further Explanation of the Interplay between Top-down and Bottom-up Reprocessing as Explained by (a) Panksepp and (b) Adolphs

(a) Panksepp The interplay between top-down and bottom-up processing holds significant implications for the treatment of trauma. Panksepp [21] characterises these as;

1. Tertiary Affects and neocortical 'Awareness' functions. This includes thoughts and planning involving prefrontal cortex, emotional rumination and regulation and free will or intention to act.

2. Secondary processes or Affective memories. This involves basal ganglia learning and comprises classical, instrumental and operant conditioning. The seeking is driven by nucleus accumbens. Finally there are unconscious emotional and behavioural habits from dorsal striatum.

3. Primary processes involving primordial affective states. This includes sensory affect including pleasure and disgust, homeostasis and emotion action systems.

According to Panksepp's theory, for higher MindBrain functions to operate, they must be integrated with lower BrainMind functions. These primary to tertiary gradients of mental development yield nested hiercharchies of interrelationships. Lower functions are rerepresented within higher ones. Thus multiple avenues of bottom up and top down reprocessing exist. He believes it is essential to elucidate the most hidden level of mental organization, the primal affective level, in order to understand the higher levels.

The crux of the problem in trauma stems from an inability to process sensory motor and emotional responses and the diversion of the frontal lobes' blood supply necessary for effective cognitive processing to supply the major organs such as heart lungs and gastrointestinal tract. Sequencing of responses are a necessary prerequisite for information processing and the pressure for completing these processes is essential at the basic foundational level of processing of sensory and motor responses. When someone experiences a traumatic event the sensory motor systems become activated and often overwhelmed in the response to threat. When these functions are overwhelmed sensory motor processing stops and further affective processing at the cortex level is blocked out not only in the moment of the trauma but also in the future. Thus the interplay between topdown and bottom up processing can hold these traumatic reactions in place.

My unique therapeutic approach is to integrate emotional sensory and cognitive processing with brainstem activation. I have called this Bilateral Affective Reprocessing of Thoughts (BART). It can also be conceived as combining trauma focused Cognitive Behaviour Therapy, the latest EMDR protocols with the newer approach of sensorimotor psychotherapy. The thalamus acts as a relay station of sensory information from the periphery to the cerebral cortex. The only exception is input from the olfactory system, which passes directly to the amygdale, which is also known as the seat of the emotions. The thalamus integrates the brain's level of consciousness, alertness, arousal and neuroplasticity. The thalamus transmits a wave impulse of 40 cycles per second throughout the brain. When traumatic memories occur they are burned into the limbic system with the associated distorted sensory information. They remained stored at this level and the thalamus is unable to relay information to the cortex. Careful sensory motor therapy integration enables the process of thalami-cortical binding to occur so that the information transfers firstly to the right hemisphere and then to the left hemisphere where it is associated with language and meaning and Broca's area. The patient can now make sense of the traumatic experience. In BART psychotherapy and peak performance the medial, lateral and orbital prefrontal cortex are crucial to engage as there is a danger that during the trauma they will be off line and unable to process relevant information.

(b) According to Adolphs, emotional processes can both begin and terminate rapidly. However this does not preclude later emotional experiences from modulating this response. Feelings may be based on bodily representations and the images in Figure represent the sequence of events schematically. Emotional responses range in time from less or equal to $100 \mathrm{~ms}$ to several minutes. Responses to emotional visual stimuli can occur rapidly in the prefrontal cortex or amygdala.

At around $100-200 \mathrm{~ms}$ post stimulus, sensory cortices provide more detailed input to parts of the limbic system eg amygdala The superior temporal cortex shown in green encodes dynamic aspects of face procession namely facial expression. The fusiform cortex shown in blue encodes static information such as identity.

Once the emotional meaning of a stimulus has been evaluated by the brain, emotional responses are triggered in the body by projections from the amygdala and prefrontal cortex to the brainstem and hypothalamus (not shown). This leads to repr sentation at the level of the insular cortex, which forms the substrate for conscious feelings such as taste and disgust.

This provides evidence for the reasons gut feelings and heartfelt sensations can be reprocessed by the brain during the continuous bilateral stimulation at the peripheral and cerebellar level during sessions of BART psychotherapy.

Thus the interplay between bottom-up and top-down information reprocessing that gives rise to an emotional state is a result of both complex and variable neural mechanisms.

Int J Psychol Behav Anal

ISSN: 2455-3867

IJPBA, an open access journal

Volume 2. 2016. 118 
Citation: O'Malley AG (2016) Beyond the Art of BART: Bilateral Affective Reprocessing of Thoughts as a Dynamic Model for Psychotherapy Across the Lifespan. Int J Psychol Behav Anal 2: 118. doi: https://doi.org/10.15344/2455-3867/2016/118

Page 9 of 15

It is essential to manage the patient's reprocessing during BART psychotherapy within calm WATERS, otherwise hyper or hypo arousal can occur (Figure 13). The resultant anxiety can trigger panic attacks and nervousness. Safely negotiating this stage allows the emergence of meaning and intuition for the patient. The patient starts to view their life with a new perspective.

Dr Giovanni Liotti discusses the links between trauma, dissociation and disorganized attachment [23]. Disorganized attachment, genetic, temperamental and neurobiological vulnerability and adverse childhood experiences are risk factors for

a. PTSD via peritraumatic dissociation

b. Complex trauma

c. DID dissociative identity disorder and

d. BPD Borderline Personality Disorder

He describes the 'drama triangle', which involves persecutor, rescuer and victim. There is a distorted internal working model of attachment. For e.g. a 6 year old will show collapse of a seemingly coherent strategy of thought, behaviour, emotion and attention when shown pictures of the Separation Anxiety Test [24]. When careful consideration is given to the role of attachment dynamics to how trauma is experienced, then the difference is not whether trauma is direct or indirect but whether traumas are inflicted by an attachment figure or by an anonymous destructive force. The dissociating potential of the former is predicted to be greater than the latter according to attachment theory.

It is my hypothesis that unprocessed affect from the attachment figure is stored in a sensorimotor state or unconsciously in the body. My experience of using BART psychotherapy is that when the patient is contained within their window of tolerance or calm WATERS, this bodily state and accompanying repressed emotion cane be activated using bilateral tactile and cerebellar stimulation. Over the $120 \mathrm{~min}$ BART session it is my clinical experience that this information is reprocessed and integrated with explicit memory, semantic meaning and self-knowledge.

Recently [25] a severe form of ADHD was observed in individuals exposed to severe deprivation in early childhood. These results were found despite these patients spending most of their lives in loving supportive and well-resourced families. The risks of developing ADHD seemed to increase with age and be related to early disturbance of critical neurobiological functioning. This cohort of Romanian adoptees also had features of ASD and difficulties with attachment relationships. These findings highlight the need to assess exposure to adverse environments in children with ADHD. There should also be an effective transition from adolescent to young adult services in ADHD patients exposed to early adversity. It is my hypothesis that BART psychotherapy provides such a therapeutic framework. This needs to be the focus of future research.

My clinical experience using this model of dissociation is that it is easily understood by patients and can help them to stay within their window of tolerance and emotional regulation during reprocessing of traumatic stress with BART psychotherapy and peak performance.

\section{Areas for Future Development into the Theory and Practice of BART Psychotherapy}

Newly discovered structural and functional features of the immune system and the central nervous system. This involves a newly discovered meningeal network of lymphatic endothelial cells provides

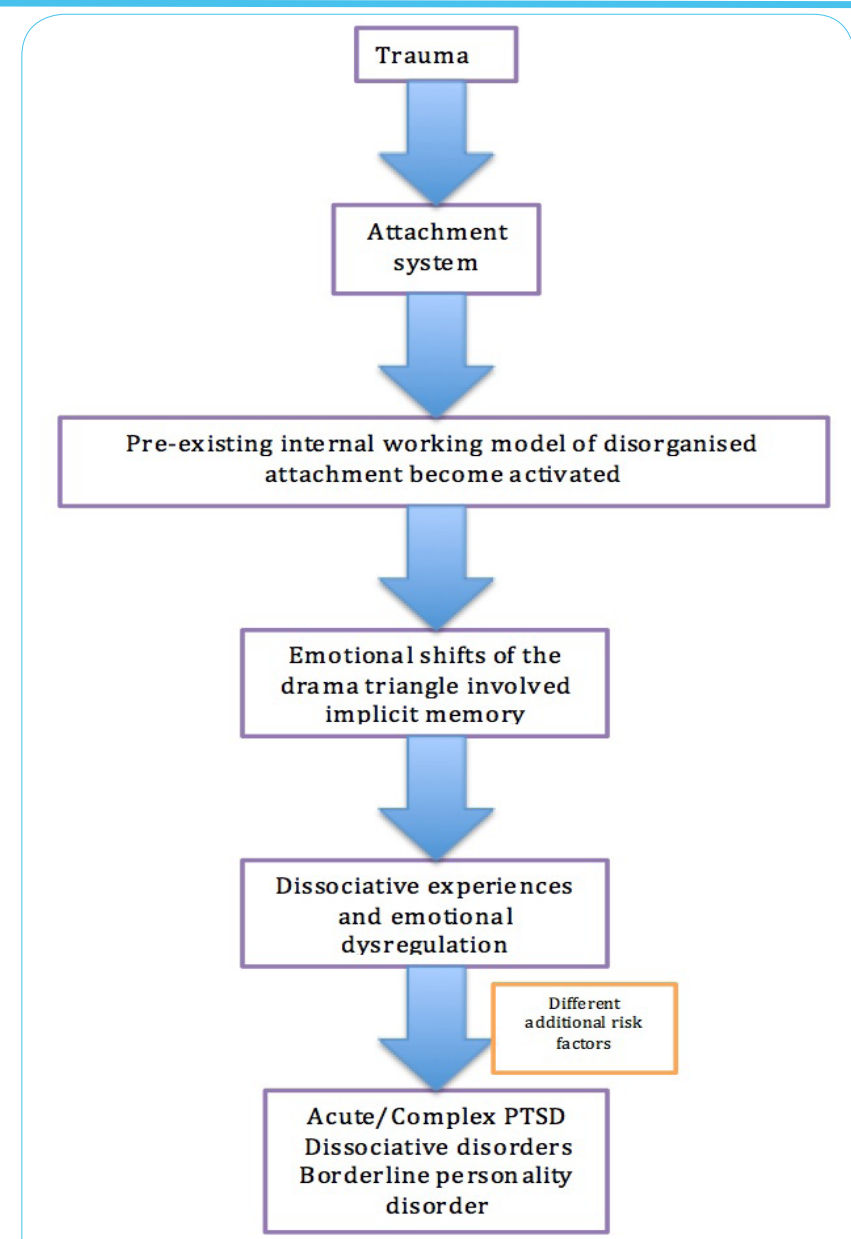

Figure 12: Model of links between trauma, attachment and dissociative disorders mediated via the Internal Working Model (IWM) of disorganized Attachment.

evidence for gutbrain heartbrain and headbrain hypothesis central to BART psychotherapy.

In recent years links between the immune system and the central nervous system have emerged. This has opened up a new vista for combined treatment of physical and mental health disorders using BART psychotherapy.

The presence of lymphatic endothelial cells in the CNS has recently been discovered, [26]. Researchers demonstrated that these endothelial cells transported fluid and immune cells from the CSF to deep cervical lymph nodes. This enables a connection to the rest of the lymphatic system namely lymph nodes, thymus, spleen and bone marrow. Lymphatic vessels were found lining the dural sinuses. The dural sinuses drain blood from both the internal and external veins of the brain into the internal jugular veins. Blood from the meningeal lymphatic network appears to start from both eyes and track above the olfactory bulb before aligning next to the sinuses. The glymphatic system is the waste clearance pathway for the CNS. The glymphatic system was so named by Maiken Nedergaard [27] a Danish neuroscientist, as it is dependent on glial cells. Now with the discovery of Louveau et al the meningeal lymphatic vesselsprovide a pathway for CSF drainage and for immune cells to be transported from the central to the peripheral nervous systems. The discovery of this CNS lymphatic system calls for reassessment of neuroimmunology fundamentals. Also disorders with an immune system dysfunction 


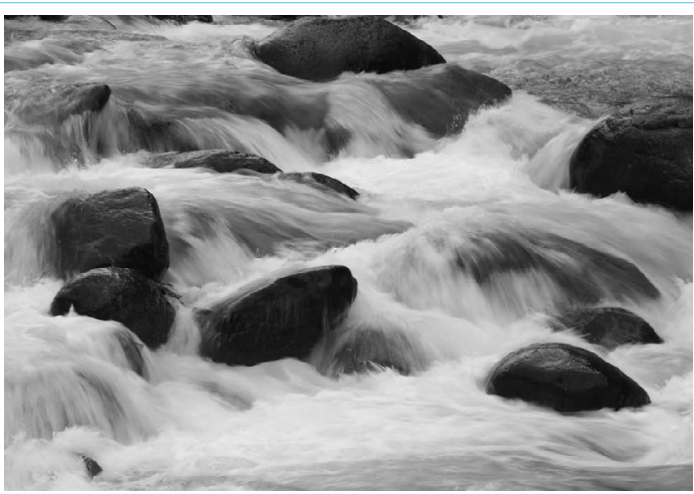

(a) Hyperarousal and dissociation.

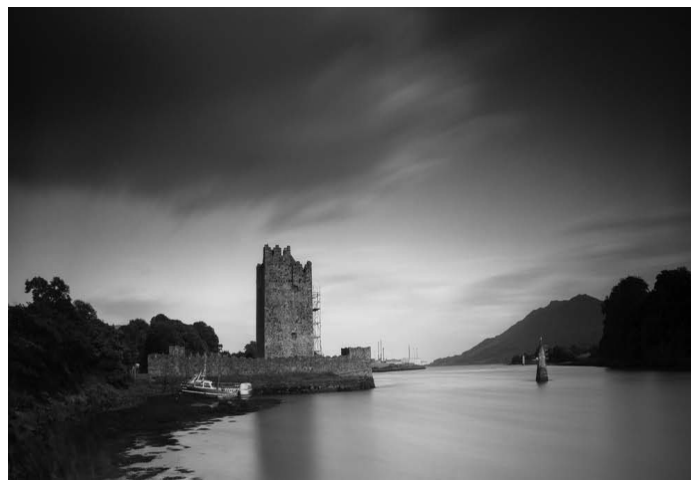

(b) Integration of affective states

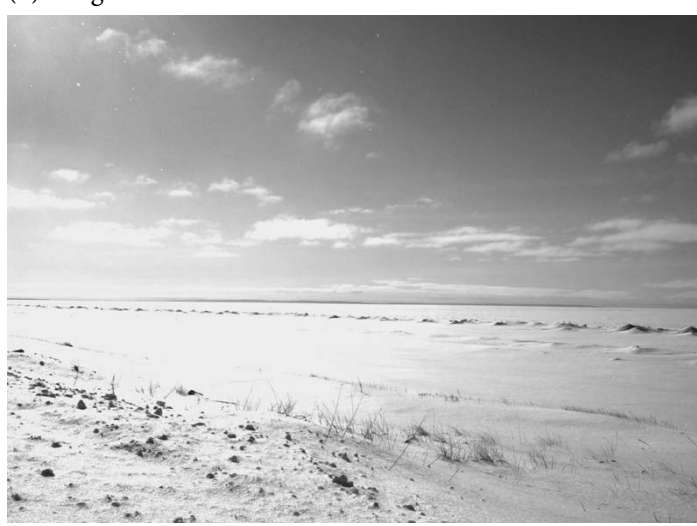

(c) Hypoarousal and Dissociation

Figure 13: Model of altered levels of arousal dissociation and stability linked to the window of affect tolerance and emotional regulation. The middle picture is of Narrow Water Castle in Warrenpoint, Co Down, Northern Ireland. This was the location for the greatest loss of life of security forces in the Troubles in Northern Ireland. It is designed to illustrate how the state of calm represented by 'Waters' can change in seconds to the destructive states of chaotic 'Rapids' and numbing 'Frozen'. This can lead clinically to the dissociative states described above.
RAPIDS Racing thoughts Affective dysregulation Partitioned personality Impulsivity Distress Suicidality

WATERS Window of Affect Tolerance

Emotions Regulated and Stabilised

FROZEN Freeze Reaction Oblivious to the outside world Zonked out Emotionally Numb may have malfunction of the meningeal lymphatic vessels as a significant aetiological factor. BART psychotherapy has the flexibility to work with other medical and surgical treatments to stimulate the nervous system to improve immune system function improving the outcome in these conditions.

Role of the immune system in the Gut or Enteric Nervous System and the implications for BART Psychotherapy. The word glia is derived from the Greek word for 'glue.' These cells act as the glue of the enteric nervous system. Enteric Glial cells (EGC's) form a neural network in the intestinal mucosa. They interconnect with somatosensory, circulatory, nervous and immune systems. Evidence suggests EGC's modulate gut functions by regulating mucosa permeability and neural activity of Meissner's and Auerbach's plexi. They also interconnect with the body's immune and endocrine systems. They are involved electrolyte secretion, absorption and vascular tone of the gut. These can all be related to processing of gut feelings or gut instinct in the initial stages of BART psychotherapy. Disease states such as infection, inflammatory bowel disease, irritable bowel syndrome, ischemic bowel, slow transit constipation e.g. in depression, diverticular disease, neurodegenerative diseases such as Parkinson's disease that decrease gut motility are all worsened by a reactive EGC phenotype.

It is my hypothesis that the therapist can initiate signalling of mirror neurons in the gut-brain axis of the patient using bilateral stimulation as described in The Art of BART [8]. This may restore 
proper EGC function and lessen the onset and progression of intestinal inflammation, which is a key component in many of the above intestinal diseases. Future research can look at how neural stimulation can impact positively on the immune system with a focus on bilateral stimulation the vagus nerve.

The thymus and lymph nods regulate cellular immunity. Bone marrow and spleen regulate humeral immunity while skin and mucosa are the first line of defence of innate immunity. T lymphocytes result from cellular immunity and B-lymphocytes from humeral immunity. The vagus or 'wandering' nerve modulates the immune system and has links to the immune system of the heart and the CNS. The nucleus tractus solitarius (NTS) is a major relay station for neural-immune communication. Use of tactile BLS on either side of the brain stem during BART psychotherapy can enhance communication between the neural networks and the immune systems. The hypothesis is that cerebellar activation at a frequency of $40-60 \mathrm{~Hz}$ (gamma waves) will dampen brain stem responses both minimising inflammation, boosting immune-neural communication and thalamocortical binding, (Figure 14).

The NTS connects to the dorsal motor nucleus of the vagus nerve and the nucleus ambiguus. The vagally mediated heart rate variability (HRV) is positively associated with activity in the anterior singulate cortex, insular cortex and amygdala. This pathway from the brain stem to the prefrontal cortex is activated in the final stages of BART psychotherapy. This neural network involves the autonomic nervous system, the endocrine system and immune systems as well as reprocessing of pain, emotion and changes in behaviour. BART psychotherapy is one of the few therapeutic approaches to take account of these multiorgan systems.

Unexpected role of interferon gamma in regulating neuronal connectivity and social behaviour, [28] provides an opportunity for further research into the efficacy of BART psychotherapy Human meningeal immunity was previously known to influence spatial learning and memory and has now been shown to affect social behaviour. Mice, whose adaptive immunity was compromised, showed enhanced connectivity of the frontal cortex and this was associated with marked deficits in their social behaviour. Interferon gamma (IF $\gamma)$ appears to mediate an adaptive immune response and promote social behaviour at the same time. Gaba amino butyric acid (GABA) is produced by inhibitory neurons that react with IF $\gamma$. The subsequent neural circuits that care recruited promote social behaviour. This confirms the link between the brain's meningeal immune system and social behaviour at a molecular level.

This also suggests a possible evolutionary link going back to the origin of life. A conflict existed between social aggregation of the social behaviour of species and an antipathogen response that is protective in situations where new species are encountered. Thus natural selection appears to have worked out that the risks of encounters with a new species (acquisition of potentially fatal diseases) is outweighed by the potential benefits in terms of survival and reproduction. For Homo sapiens this involves the promotion of mental health, consequent to adaptive social behaviour resulting from closer contact between species.

Many neurological and psychiatric disorders show disturbance in social behaviour e.g. ASD, ADHD, OCD, dementia and schizophrenia. These are also linked to immune system dysfunction for example altered levels of cytokines, $\mathrm{T}$ lymphocytes and natural killer cells. This discovery many help to elucidate how immune system dysfunctions

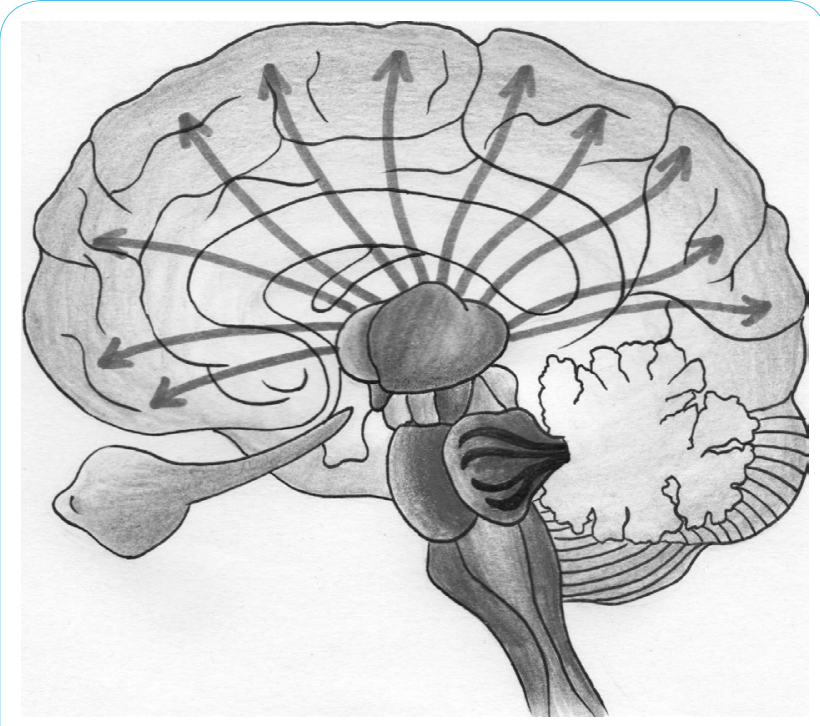

Figure 14: The cortex under the influence of an optimally resonant thalamus. This can occur during BART psychotherapy as a result of thalamocortical binding. The connections of the cerebellum to the corpora quadragemina are illustrated. Continuous bilateral cerebellar stimulation during BART psychotherapy helps to dampen instinctive levels of hyperarousal experienced at a brainstem level in the aftermath of trauma. The further connections between the thalamus and all areas of the association cerebral cortex are also illustrative of the impact BART psychotherapy is postulated to have on learning from these events via a reflective capacity and development of insight. Drawn by Sorcha O’Malley.

disrupt neural circuitry responsible for social behaviour in these disorders.

BART psychotherapy has now been modified in light of this recent scientific breakthrough to develop protocols to attempt stimulation of improved immune neural communication. It is now clear that the brains of the head, heart and gut interact to achieve integrated information processing at all levels of hormonal, circulatory, central, cardiac and enteric nervous systems. Recent discoveries show a network of connections between the lymphatic meningeal pathway of the brain and the normal immune system. As far as I am aware BART psychotherapy is the first comprehensive therapeutic approach to incorporate this knowledge, which was recently published, [26].

Patients with ASD show hyper-connectivity in the prefrontal cortex and insular regions of the frontal lobe. CNS neurons respond to IF $\gamma$, which comes from $\mathrm{T}$ lymphocytes, which produce this inhibitory neurotransmitter GABA. The activated inhibitory interneurons act to prevent hyper-excitability and connectivity in the prefrontal cortex. Thus abnormal immune responses may be the root cause of many neurological and psychiatric disorders presenting with deficits in social behaviour. These pathways between the nervous and immune systems have evolved slowly over millions of years. Consequently they may be especially vulnerable to fast evolving pathogens such as those causing meningitis and the Zika virus. It was previously thought that the brain was immune privileged, this research shows it to be immune competent. The activation of imagination during BART psychotherapy may not only stimulate mirror neuron activity but also enhance immune-neural communication at the level of multiple body systems. This can be a subject of future research. 


\section{Conclusions and Future Directions for BART Psychotherapy}

Prof Kevin Tracey, [24] stated:

"Neuroscience and immunology are now armed with the new paradigm that specific steps in immunity are modulated by discrete neural circuits that signal through defined molecular mechanisms."

It is likely that further links will be discovered. With BART psychotherapy incorporating this new scientific paradigm can be accomplished from either a top-down or a bottom-up approach. This is because patient reflexes and responses require both afferent signals from the interaction of the gut and heart brains with the efferent signals from the immune and nervous systems. Also efferent messages to the enteric and cardiac nervous systems from the nervous and immune systems can be activated using the alternating frequencies of tactile and cerebellar and cortical resonance. BART psychotherapy continually monitors the two-way interaction of information reprocessing between the mind and body during the session. In moving Beyond the Art of BART, I believe use of the buzzers as I have described, will both Activate and Reprogram Thoughts and help the client Reorganize Tasks. Mapping these circuits anatomically and functionally is likely to reveal important information about the network of information sharing between two elaborate, highly coevolved systems of defense and memory. This is a key goal of research into the effects on the Default mode Network of Bart psychotherapy pre and post treatment.

\section{Feedback from patients}

The following examples provide practical examples of the use of BART and qualitative accounts or patient reported outcome measures (PROMs)

\section{Case example 1}

A heavy goods vehicle driver was referred to me following involvement in a fatal road traffic accident several years earlier. Since then he had attended more than 90 individual sessions of CBT. He spoke of how he dreaded these sessions as he was often asked to repeat the history. This became increasingly difficult because of his dysregulated affect. He was in a state of speechless terror and experienced continuous fear and hopelessness. This had led to severe depression with suicidal intent. During BART psychotherapy I used bilateral cerebellar stimulation to regulate his affect. I asked him to imagine the chair as the driving seat in his heavy goods vehicle. I asked him to hold the bilaterally activated buzzers in each hand as if holding onto the steering wheel. I used the freeze frame technique to slow the reprocessing into manageable images. His peripheral nervous system became reactivated as if he was reliving the event in vivo. His grip tightened on the tactile buzzers and as re revisualized the point of impact his right leg shot forward as he sat in the chair. My patient had his eyes closed and was unaware of this instinctive motor impulse.

When I drew his attention to the sudden movement, he realised this was when he applied maximum pressure to the brakes of his heavy goods vehicle. Metaphorically it was as if his life had been put on hold since the accident as at a somatomotor level he was still applying the brakes to his life in an unconscious way. During the BART psychotherapy session he was encouraged to mindfully let go of these 'brakes' in order to let his life 'get back on the road'. This stoic patient was able to relive the experience and grieve appropriately realising that the driver who swerved into his path was the guilty party and that the accident was not his fault. The physical injuries he suffered from the accident and the associated traumatic images of the driver of the other vehicle were readily dealt with during several further sessions of BART psychotherapy. His latest feedback was that he had recovered his previous zest for life and was well on the road to recovery. The key learning point from this case is that the stuckness in processing was due to an unconscious movement impulse that had literally put the brakes on this patient's life preventing him from moving forward Identification of this and then mindfully allowing the patient to become conscious of this movement helped to lift his foot of the brakes and start to gently press on the accelerator pedal again.

\section{Case example 2}

A patient had experienced numerous physical health problems culminating with diverticulitis and was at risk of developing peritonitis. My new intensive model of BART psychotherapy involved her attending for 15 hours of BART psychotherapy spaced out over 3 days. The feedback was so positive that she stated the detailed and in depth work had brought her more insight than the ten years of psychotherapy she had received previously. Her written feedback was as follows:

"In my session I was working on my recent traumatic experiences involving repeated infection, misdiagnosis and eventual development of septicaemia on two occasions. The infections left damage to the bowel resulting in ongoing symptoms and in addition a recent diagnosis of diverticulitis. I was focusing on my experience of these events particularly the sense of not having a voice and not been heard throughout numerous assessments; I had felt very unsafe, helpless and uncared for throughout that time. I then focused specifically on the bowel symptoms, using the bilateral activation and was being guided through the "light stream" exercise by Art and had reached a stage in which I was imaging harnessing a source energy that provided a violet healing light; during this visualisation work I became aware of my father joining me to offer support and love - he died more than 30 years ago from peritonitis arising from a bowel rupture related to diverticulitis from which he had suffered for about 10 years. My eyes were closed but I "saw" him sitting to my left, slightly behind me with his right arm encompassing me. He emanated a powerful golden light from his upper and lower body and I could "see" a small section of his jacket around the righthand side pocket - a jacket I recall him sometimes wearing in the last couple of years of his life. The light was infinitely more powerful that the one I was visualising. . I "heard" him say, "that I did not need to suffer as he had done". My sense of his presence and communication from him was, as real as if I was seeing, feeling and hearing his presence but it did not involve any of the senses that relate to the outside world. It never occurred to me to open my eyes or to turn to my left to see him more clearly but the experience was absolutely as real. I could not answer the question as to how I knew it was he but I knew with the same certainty and recognition I would experience if my son or daughter suddenly joined me in a room in real life.

I experienced this and continue to experience it as a most ordinary event; my Dad just dropped by to help and yet my conscious mind sees it as an extraordinary event for which I have no rational explanation. I experienced a profound sense of safety in his presence; this is a novel feeling for me having grown up in a very insecure, unpredictable environment related to my father's alcoholism, which manifested in extreme binges every few months and my mother's untreated complex PTSD. The sense of safety has remained with me, not in the sense of a profound new experience as occurred when my father was with me 
but in an ordinary sense of it now being possible for me to live in the present in a way that was never previously possible. This has resulted in a new sense of vitality and pleasure in life."

This shows how BART psychotherapy is a therapeutic approach that taps into a powerful source of healing at the deepest level of connection the patient can achieve with their loved ones.

I also treated her 21-year-old son who has dyslexia and severe social anxiety. He had failed to socialise during his first year of university abroad and mum was concerned for his future. He also tended to have difficulty with organization and completing assignments partly due to his dyslexia.

\section{Recent feedback is as follows:}

"He seems to be really making strides. He has been out a couple oftimes each week with various friends and it seems to be an 'ordinary' event for him rather than a major anxiety provoking possibility. His work seems to be going very well and he sounds much more organized than ever in the past. Also, he is trying new things and has just been rock climbing, not easy with size 13 feet! But he was very pleased that he did well at it, which is interesting as his balance and coordination were extremely poor as a child but that seems to have shifted. He sounds very happy with life at present."

\section{Case example 3}

My name is Aggeliki Pappa and I am the founder and head director of studies of 'I love dyslexia' language school, the first and only school in the world, highly specialized for teaching English as a foreign language to students with dyslexia and special educational needs in a holistic way. Our innovative school was established in 2009, and since then we have received numerous awards and we organize events under the auspices of UNESCO, as the Hellenic National Commission for UNESCO has nominated I love dyslexia for Hamdan Prize. Personally, duo to my pedagogical innovation and its results, I found myself in the top 50 educators in the world for the Global Teacher Prize 2016 and I am presenting I love dyslexia holistic methodology that stands for sustainable development, next month in United Nations Geneva, Peace week. I am the leader of the literacy group of the Varkey Foundation, collaborating with Dr Reimers in Harvard University and I am Reseacher and teacher trainer in many European projects with Folkuniversitetet, Uppsala. I love dyslexia holistic method, which shares common ground and principals with BART model in educational context, received support and verification on its neuroscientific verification from Dr Theoharidis from Tufts University, while I am now collaborating with Birmingham University, graduate school of Education, designing a module in a new Master degree with Dr Reraki, where teachers will be trained on I love dyslexia approach. I had the honour to meet in person Dr O Malley in London, where we both presented in a Conference. I was really impressed by his innovative model of intervention and I immediately associated it with the innovative intervention we implement in I love dyslexia school, and has very positive and transformative results to our students. Later on during our common days I London, I had a Psychotherapy session with Dr O Malley, and it was a wonderful experience for me. I felt relieved and empowered as well as a sense of enlightenment. It felt like something worked in my subconscious level, and day after day after our session, I feel more relaxed, less nervous, with a wonderful positive sense leading my daily life. I believe that Dr O Malley's holistic, innovative approach of intervention has opened a new door to the field of psychotherapy as well as to holistic educational intervention, that has a lot to offer to humanity and the sustainability in the world. As a person, Dr O' Malley is one of the most virtuous, cultivated, luminous and charismatic people I have ever met, who in a very discreet and gentle way, communicates his personal and professional unique insight. I am so impressed by the results of his work on me, his deep knowledge on his field and the common ground we have in our innovative holistic approaches, and I know that is just the beginning of our collaboration, as we have already discussed the next steps of our professional common actions. Dr O Malley's model is based on the still quite unexplored power of the right hemisphere of the brain and that is why it is so unique in its nature and results. I am very grateful to have met such a charismatic and innovative professional in his field. I firmly believe the world will benefit a lot from his special talents and distinctive work. The main reason for discussing this case is that it provides an opportunity to extend BART psychotherapy into the education sector.

\section{Case example 4}

This 62-year-old male patient works as an integrative psychotherapist and is trained in EMDR. He agreed to the use of bilateral cerebellar stimulation using headphones placed over the mastoid processes and while also holding buzzers in each hand, which were activated continuously during our 2-hour sessions.

In session 1 we started with the memory of a workshop which re-enacted birth memories. Although 30 years ago this therapeutic session was recalled as activating a sense of betrayal. The session ended with a clearer understanding of what his parents had to endure caring for an infant sibling who was chronically ill for years after my patient was born. He could appreciate how he had to fend for himself.

In session 2 and $3 \mathrm{He}$ continued to resolve deep seated emotional issues and connected to the powerful memory of a neighbour who had valued him as an individual in his own right age 10. We were able to transpose this figure as an alternative attachment figure and resource for present day issues.

The key point about this work is that it has been completed entirely over the Internet although we are planning face-to-face sessions in the future I contacted Dr O'Malley to look for help with some very long standing difficulties in the area of attachment. Although now in my early 60s, I still carried some strong negative beliefs arising from my interpretation of events that I remembered in my family of origin. I also held some strong feelings around how I interpreted my own birth and around the subsequent 5 years of my life. Although this might appear to be fantasy, I had carried a strong belief that I was 'made' to be born without being included in the decision and that subsequently I resented my mother for this experience of being 'expelled' from the safety of the womb.

However, through my work with Dr O'Malley I am now able to put these 'experiences' in context. When I was born, I was the third child. My older brother, who is two years older than me, was seriously ill and remained so for a number of years. It seems clear to me know that my mother was so concerned with his health that I was allowed and/or encouraged to develop a very early independence which I interpreted through a lens of resentment. I think that by nature I tend towards independence and exploration of the limits of work and play and can now make 'peace' with my internalised 'mother'. We now support each other and are also able to take time apart. A significant moment in my 
Citation: O'Malley AG (2016) Beyond the Art of BART: Bilateral Affective Reprocessing of Thoughts as a Dynamic Model for Psychotherapy Across the Lifespan. Int J Psychol Behav Anal 2: 118. doi: https://doi.org/10.15344/2455-3867/2016/118

Page 14 of 15

therapy with Dr O’Malley came when I was able to say to my mother: 'I've been away' (from her), to which she replied: 'so have I'.

I look forward to continuing this work.

\section{Case example 5}

This 60-year-old patient had 13 -hour session, which dealt with the following issues:

1. When age 8 she had to leave the family home with her mother to escape domestic violence by her father against her mother.

2. Memories age 5 when she had a cough and was told to be quiet by her mother so as not to upset her father. Aware of the abuse her mother was likely to be on the receiving end she almost suffocated herself with a pillow in an effort to keep quiet and sleep.

3. Multiple episodes of child sexual abuse from age 8 to 11 from a neighbour who used to babysit. The buzzers where used to activate a sense of healing and I user the metaphor of using the buzzers as a taser to force him away from her.

"I visited Dr O'Malley two weeks ago to have a consultation with him and to take part in BART therapy. The session was professionally led and I felt fully supported both during and after the session. Whilst experiencing this therapy, I accessed memories and emotions, which were in a deeper level than I have previously experiencing. The session was quite challenging and I became very emotional on a couple of occasions, but I felt safe and in a supportive environment. I am a hypnotherapist and complementary therapist and have had psychological intervention in the past. Following the session of BART I had with Dr O'Malley I went through a number of experiences. For a number of nights after the session I had very vivid dreams, and although they were not disturbing, they were thought provoking. Two days after the session, I was so tired I felt that I could hardly move which lasted 12 hours. Four days after the session I had a very heavy cold, most unusual for me, with my nose just streaming and a headache. I am no stranger to healing crisis reactions from physical therapies I have had, but have never had this reaction from psychological therapy. My relationship with my husband of 37 years can be stormy at times and since the session I have been interacting with him on a different level. Previously I would of been afraid of confrontation and raised voices, my husband has a very loud voice and this has bothered me in the past. I have barely noticed it since, and have felt closer to him. There has been a communication issue for us as a couple for some time too and we have had some lengthy and intimate discussions over the last two weeks. I also feel able to better stand up for myself with people who I have previously perceived as difficult and demanding. I am feeling much more at peace with the world in general with myself."

\section{Case example 4}

This 25-year-old patient presented with social phobia and anxious attachment. This was related to a severe bout of gastroenteritis, which developed while on holiday with her parents in India age 21 months. She required resuscitation and rapid intravenous hydration and antibiotic therapy. She then was rapidly discharged and flown to Europe to prevent acquiring further infections to which her depleted immune system would have succumbed. This was probably involved in her Aetiology of clinical hypothyroidism for which she was on daily thyroxine. Some of her feedback was as follows:
I was asked to describe two experiences from my time with this therapy. The therapy was intensive, spanning over the course of three days and I was very resistant to the whole process at the start.

I do not like therapy in any form. I'm very sceptical of new forms of it. I had a bad experience with it once and closed the door on the whole affair. But my mother was insistent so I went along to appease her.

It was slow going at first and it wasn't until the second day when I had a session alone - as opposed to with my mother - that I actually saw why my mother and brother were so positive about it.

Well, I felt more than saw.

I don't remember what we were actually discussing at the time but it was as if something changed.

Picture this. A lightweight fabric, like a cross between a chiffon and silk, settling just above the elbows and it unravels at great speed all the way up to the temples, not quite touching the skin but there. If there's warmth to it, it's very mild but it has lightness to it. Almost like lightheadedness but far more pleasant.

I described it as something like a freeway from the body to the head.

Emotions, feelings, anything like that struggled to pass between my head and body -I can understand that, I have defensive walls about twelve inches thick - but I guess the emotional progression was being forced to move along with the vibrational buzzers and, eventually, if they couldn't find a way internally, they found a way externally, through the 'aura' or mental mapping or whatever it might be.

This reaction - because it was so vivid and undeniable (I really did feel something after all) - is the only reason I opened up to this process at all.

\section{Because I felt - physically felt - it was actually doing something.}

And I honestly believe it really did do something because each time I look back to have a hunt around for those negative feelings we had worked to dispel, I can't find them.

It's like I have a memory of them, I know they were there once, but that's it. The same way I know I broke a toe once, I remember it hurt but the pain isn't actually there anymore.

As it turned out - and this was the other thing I was asked to describe - that sudden lack of negativity is actually quite frightening.

It sounds bizarre, I know. Who could be frightened of being rid of painful, ugly emotions? But it is scary because I had been carrying around those emotions for years, I had built defences against them and they had been there for such a long time, like a wet blanket I dragged around with me.

And then suddenly they weren't there anymore.

It was just a matter of adjusting.

This is certainly the only therapy, outside of traditional, that I would recommend to friends, simply because I can guarantee that, at least for me, that it worked. And it's the only therapy I would go back to if I needed to deal with things.

More than that, it's a therapy that a person can take home with them, as in the techniques. I can take what I learnt from the sessions like noting where feelings sit in the body - and work within myself to move them along, process them and remove them. 
Citation: O'Malley AG (2016) Beyond the Art of BART: Bilateral Affective Reprocessing of Thoughts as a Dynamic Model for Psychotherapy Across the Lifespan. Int J Psychol Behav Anal 2: 118. doi: https://doi.org/10.15344/2455-3867/2016/118

Page 15 of 15

Some implications for treatment of patients with symptoms of traumatic stress during sessions of BART psychotherapy

The patient may show 'stuckness' in trauma processing via a movement impulse as in the first example, a physical sensation, a gut feeling, reaction or instinct, a core belief or intense affect or destructive thought process. Individual therapies that focus on one or two of these may miss the vital factor causing the blockage to information processing. In the later stages of BART psychotherapy, wider chains of association to the original trauma are made with the patient. This allows any residual pockets of trauma to be located so that they can be actively reprocessed towards resolution and recovery. The patients usually respond by saying they feel lighter and, "It is as if a weight has been lifted from my shoulders". I encourage the patient to notice further reprocessing that may occur after the session. Thisoften comes in the form of a new insight or dream moving on from the original traumatic experience. I encourage patients to write this down and or email me so we can discuss the meaning for them at the start of the next session. Thus Rapid Eye Movement or REM processing continues for days and weeks after face-to-face therapy has ended. The cerebral cortex has space freed up to learn from and attribute meaning to their therapeutic experience. In a sense they gain a deeper level of insight.

\section{Results}

More than 200 families have received BART psychotherapy at intervals of weekly, twice weekly or intensively (20 hours over 3 days). The most positive feedback has come from those families receiving intensive treatment over 3 days. This seems to allow cumulative gains to be made in resolving issues each day and the scores on the impact of events scale have returned to normal. The scientific collation of this case based information is a goal for a future article.

\section{Declaration of Interests}

I have written the book The Art of BART published by Karnac Books in 2015 and developed a machine, which assists the therapist in delivering this intervention.

\section{Acknowledgements}

I would like to thank Clíodhna O’Malley and Pat Scully of Redacteurs Ltd for their immense patience in helping with the preparation of this manuscript and Sorcha O'Malley whoprepared all the unattributed diagrams and drawings.

\section{References}

1. Darwin C, Darwin PC (2007) The expression of the emotions in man and animals. United States: Biblio Bazaar

2. Langley JN (1921) The autonomic nervous system: Part 1. Cambridge, England: W Heffer, Located at: Cornell University Library.

3. Hess W (1949) Nobel Prize.

4. Porges SW (1995) Orienting in a defensive world: Mammalian modifications of our evolutionary heritage. A Polyvagal theory. Psychophysiology 32: 301-318

5. Porges SW (2003) Social engagement and attachment: a phylogenetic perspective. Ann N Y Acad Sci 1008: 31-47.

6. Porges SW () The polyvagal theory: New insights into adaptive reactions of the autonomic nervous system. Cleve Clin J Med 76: S86-90.

7. Porges SW, Doussard-Roosevelt JA, Stifter CA, McClenny BD, Riniolo TC (1999) Sleep state and vagal regulation of heart period patterns in the human newborn: an extension of the polyvagal theory. Psychophysiology 36: 14-21.
8. O'Malley A (2015) The Art of BART - Bilateral Affective Reprocessing of Thoughts as a Dynamic Model for Psychotherapy (1st edition), The Studio Publishing Services Ltd., editor. London: Karnac Books.

9. Jackson JH (1958) Evolution and Dissolution of the Nervous System in Selected Writings of John Hughlings Jackson. Taylor J, editor. New York: Basic Books.

10. Cheng Z, Powley TL (2000) Nucleus ambiguus projections to cardiac ganglia of rat atria: An anterograde tracing study. The Journal of Comparative Neurology 424: 588- 606.

11. Canli T, Desmond JE, Zhao Z, Glover G, Gabrieli JD (1998) Hemispheric asymmetry for emotional stimuli detected with fMRI. Neuroreport 9: 32333239.

12. Fox NA, Kimmerly NL, Schafer WD (1991) Attachment to mother/ attachment to father: a meta-analysis. Child Dev 62: 210-225.

13. Noesselt T, Driver J, Heinze HJ, Dolan R (2005) Asymmetrical activation in the human brain during processing of fearful faces. Curr Biol 15: 424-429.

14. Simon-Thomas ER, Role KO, Knight RT (2005) Behavioral and electrophysiological evidence of a right hemisphere bias for the influence of negative emotion on higher cognition. J Cogn Neurosci 17: 518-529.

15. Kis A, Huber L, Wilkinson A (2015) Social learning by imitation in a reptile (Pogona vitticeps). Anim Cogn 18: 325-331.

16. Ogden P (2007) Sensorimotor Psychotherapy Training for the Treatment of Trauma Manual and Workbooks. Boulder Colorado: Sensorimotor Psychotherapy Institute.

17. Hobson AJ (1995) The chemistry of conscious states: How the brain changes its mind. Boston, MA, United States: Little, Brown \& Company.

18. Hannaford C (1995) Smart moves: Why learning is not all in your head. 2nd ed. Arlington, Virg.: Great Ocean Publishers.

19. Given BK (2002) Teaching to the brain's natural learning systems. Alexandria, VA, United States: Association for Supervision \& Curriculum Development.

20. O'Malley A (2011) The Art of BART. Montreal: ISSTD.

21. Tsuchiya N, Adolphs R (2007) Emotion and consciousness. Trends Cogn Sci 11: 158-167.

22. Panksepp J, Asma S, Curran G, Gabriel R, Grief T (2012) The Philosophical Implications of Affective Neuroscience. Journal of Consciousness Studies 19: 46-48.

23. Liotti G (2004) Trauma Dissociation and disorganized attachment: Three strands of a single braid. Psychotherapy: theory research practice training 41: 472-486.

24. Main M, Kaplan N, Cassidy J (1985) Security in infancy childhood and adulthood: a move to the level of representation. Monographs of the society for research in Child Development 50: 66-104.

25. Kennedy M, Kreppner J, Knights N, Kumsta R, Maughan B, et al. (2016) Early severe institutional deprivation is associated with a persistent variant of adult attention-deficit/ hyperactivity disorder: clinical presentation, developmental continuities and life circumstances in the English and Romanian Adoptees Study. Journal of Child Psychology and Psychiatry 57: $1113-1125$.

26. Louveau A, Smirnov I, Keyes TJ, Eccles JD, Rouhani SJ, et al. (2015) Structural and functional features of central nervous system lymphatic vessels. Nature 523: 337-341.

27. Jessen NA, Munk AS2, Lundgaard 12, Nedergaard M2 (2015) The Glymphatic System: A Beginner's Guide. Neurochem Res 40: 2583-2599.

28. Filiano AJ, Xu Y, Tustison NJ, Marsh RL, Baker W, et al. (2016) Unexpected role of interferon-? in regulating neuronal connectivity and social behaviour. Nature 535: 425-429.

29. Tracey KJ (2002) The inflammatory reflex. Nature 420: 853-859. 